Ann. Zootech., I968, 17 (4), 469-470.

\title{
L'HÉRITABILITÉ DU POIDS A HUIT SEMAINES DE POULETS DANS LES LOTS A MEILLEURE ET A MOINS BONNE CROISSANCE ISSUS DES MEMES PARENTS
}

\author{
P. MÉRAT \\ Station centrale de Génétique animale, \\ Centre national de Recherches zootechniques, 78-Jouy-en-Josas \\ Institut national de la Recherche agronomique
}

Sur 8 générations d'une population expérimentale "synthétique " (" Jouy "), 4 générations d'une souche sélectionnée pour la ponte au domaine du Magneraud (" M I I ") et une d'une souche de RhodeIsland, l'héritabilité du poids à 8 semaines a été calculée par décomposition de la variance intragénération pour chaque sexe, d'une part sur l'éclosion ayant donné le poids moyen le plus élevé, d'autre part sur le lot de poids moyen le plus faible. Les effectifs étaient de l'ordre de 200 descendants de chaque sexe par groupe et génération avec Io à I 5 pères et 50 à 100 mères utilisées. L'héritabilité était estimée à partir de la composante "mère " de la variance $\left(\begin{array}{ll}h & 2 \\ D\end{array}\right)$, de la composante "père " (h⿻ (h) et de la moyenne des deux.

Au total, la variance phénotypique est plus grande dans les lots à croissance inférieure, tant chez les mâles que chez les femelles. Ceci rejoint des observations faites à diverses reprises par d'autres chercheurs. Quant aux héritabilités, vu l'erreur d'échantillonnage considérable pour chaque génération isolée, nous indiquons seulement leurs moyennes non pondérées sur l'ensemble des cheptels étudiés (tabl. r).

\section{TABLEAU I}

Héritabilités du poids à huit semaines dans les lots à meilleure et à moins bonne croissance issus des mêmes parents

(Moyenne sur treize cheptels)

\begin{tabular}{|c|c|c|c|c|c|c|}
\hline & \multicolumn{3}{|c|}{ Lots " lourds" } & \multicolumn{3}{|c|}{ Lots " légers" } \\
\hline & $h^{2} \mathbf{D}$ & $h^{2} \mathrm{~s}$ & $h^{2}$ & $h^{2} \mathrm{D}$ & $h^{2} \mathrm{~s}$ & $h^{2}$ \\
\hline$\delta \ldots$ & 1,43 & 0,21 & 0,82 & 0,80 & 0,28 & 0,54 \\
\hline q.. & 1,01 & 0,32 & 0,67 & 0,62 & 0,42 & 0,52 \\
\hline
\end{tabular}


On remarque l'importance de la composante "mère " dans ces estimations faites lot par lot: l'héritabilité correspondante $\left(\begin{array}{l}h \\ 1\end{array}\right)$ est plus élevée que celle rencontrée dans les estimations habituelles (1). D'autre part, dans les deux sexes, sa valeur paraît nettement plus grande dans les lots dont le poids moyen est le plus grand (lots "lourds") que dans les autres. Il faut noter que $h^{2}$ s, par contre, est légèrement supérieur dans les lots « légers " mais la différence n'est vraisemblablement pas assez importante pour être significative, du fait de l'erreur d'échantillonnage considérable attachée à ce paramètre.

Par contre, dans les générations correspondantes de la souche "Jouy ", les héritabilités obtenues à partir de la régression de la moyenne des enfants sur la moyenne des deux parents (KEMPTHORNE et TANDON, 1953) ne semblent pas différer de façon nette suivant la croissance du lot dont sont issus les premiers. La moyenne non pondérée de ces héritabilités est, chez les mâles, o,35 pour les lots "lourds ", o,46 pour les lots " légers "; les valeurs correspondantes chez les femelles étant respectivement 0,46 et 0,3 .

Par ailleurs, dans l'ensemble, on n'observe pas sur ces données d'interaction significative entre famille et lot d'éclosion.

Ces résultats suggèrent une variance " environnement " moins élevée dans les lots à meilleure croissance moyenne, en même temps qu'une plus grande importance relative des effets maternels, à en juger par les valeurs très élevées, dépassant $\mathbf{I}$, de $h_{\mathrm{D}}^{2}$ dans ce cas.

\title{
Reçu pour publication en septembre I 968.
}

\section{SUMMARY}

\author{
HERITABILITY OF EIGHT WEEK WEIGIT IN HATCHES OF CHICKENS \\ FROM THE SAME PARENTS WITII THE IIIGHEST AND LOWEST GROWTH RATES
}

On several generations of 3 strains, the heritability of 8 -week weight was estimated for each year from the "sire " $\left(h^{2} \mathrm{~s}\right)$ and "dam " $\left(h^{2}\right.$ v) components of variance, separately in the hatch with the highest weight gain and in the hatch with the poorest.

The mean value of $h_{\mathrm{D}}{ }^{2}$ for the "heavier " hatches from all flocks is very high (more than I in each sex) and higher than the corresponding value for the "lighter " hatches. On the other hand, there is no great difference between these two groups for the heritability estimated from parent-offspring regression, and no significant family $\times$ hatch interaction within generations. These results suggest a lower environmental variance and a greater relative importance of maternal effects in hatches with the best average growth rate.

\section{RÉFÉRENCE BIBLIOGRAPHIQUE}

Kempthorne O., TAndon O. B., I953. The estimation of heritability by regression of offspring on parent. Biometrics, 9, 90-100.

( $\left.{ }^{1}\right)$ On retrouve également le fait, observé par d'autres chercheurs (p. ex. RouviER et RICARD, communication personnelle) que $h_{\mathrm{D}}^{2}$ est plus grand chez les mâles que chez les femelles et que, par contre, la valeur de $h \mathrm{~S}^{2}$ est plus élevée chez les femelles, ce qui suggère l'existence d'une variance génétique liée au sexe. 